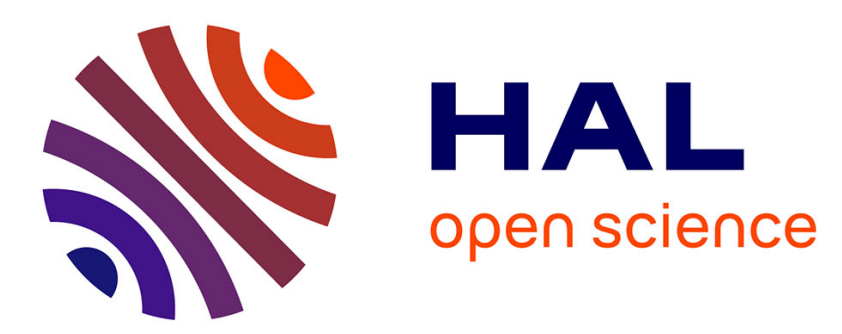

\title{
Momentum Transfer Between an Atmospheric and an Oceanic Layer at the Synoptic and the Mesoscale: An Idealized Numerical Study
}

\author{
Aimie Moulin, Achim Wirth
}

\section{- To cite this version:}

Aimie Moulin, Achim Wirth. Momentum Transfer Between an Atmospheric and an Oceanic Layer at the Synoptic and the Mesoscale: An Idealized Numerical Study. Boundary-Layer Meteorology, 2016, 160 (3), pp.551-568. 10.1007/s10546-016-0153-x . hal-01359024

\author{
HAL Id: hal-01359024 \\ https://hal.science/hal-01359024
}

Submitted on 15 Feb 2018

HAL is a multi-disciplinary open access archive for the deposit and dissemination of scientific research documents, whether they are published or not. The documents may come from teaching and research institutions in France or abroad, or from public or private research centers.
L'archive ouverte pluridisciplinaire HAL, est destinée au dépôt et à la diffusion de documents scientifiques de niveau recherche, publiés ou non, émanant des établissements d'enseignement et de recherche français ou étrangers, des laboratoires publics ou privés. 


\title{
Momentum transfer between an atmospheric and an oceanic layer at the synoptic and the mesoscale: an idealised numerical study
}

\author{
A. Moulin • A. Wirth
}

6 Received: date / Accepted: date

\begin{abstract}
The air-sea interaction at the (atmospheric) synoptic and the mesoscale due to momentum transfer only, is considered. Two superposed one-layer fineresolution shallow-water models are numerically integrated. The upper layer represents the atmosphere and the lower layer the ocean. The frictional force between the two-layers is implemented using the quadratic drag law. Experiments with different values of the surface friction drag coefficient are performed. The actual energy loss of the atmosphere and the energy gain by the ocean, due to the interfacial shear, is determined and compared to the estimates based on average speeds. The correlation between the vorticity in the atmosphere and the ocean is determined. Results differ from previous investigations where the exchange of momentum was considered at basin scale. It is shown that the ocean has a passive role, absorbing kinetic energy at nearly all times and locations. Our results show that the energy input to the ocean increases almost quadratically with the value of the drag coefficient. Due to the feeble velocities in the ocean, the energy transfer depends only weakly on the ocean velocity. The ocean dynamics leaves nevertheless its imprint in the atmospheric dynamics leading to a quenched disordered state of the atmosphere-ocean system, for the highest value of the friction coefficient considered. This finding questions the ergodic hypothesis for the idealised configuration studied here. The ergodic hypothesis is at the basis of a large number of experimental, observational and numerical results in ocean, atmosphere and climate dynamics.
\end{abstract}

Keywords air-sea interaction $\cdot$ numerical modelling $\cdot$ turbulence

Aimie Moulin

LEGI, CNRS UMR 5519, BP 53, 38041 Grenoble Cedex 9, France

E-mail: aimie.moulin@legi.grenoble-inp.fr

Achim Wirth

LEGI, CNRS UMR 5519, BP 53, 38041 Grenoble Cedex 9, France 


\section{Introduction}

Today's theory of the basin-scale ocean dynamics is based on the conservation and fluxes of (potential) vorticity (see e.g., Vallis (2006)). The large scale features of the ocean dynamics can be explained to leading order by a windstress forcing that is averaged in space and time. In numerical simulations of the ocean dynamics, performed during the past four decades, the wind-stress field was commonly averaged over one month in time and a few degrees in both longitude and latitude. There has more recently been a growing interest in the ocean circulation kinetic energy: reservoirs, sources and sinks (Ferrari and Wunsch 2009). The major input of mechanical energy into the ocean is through the wind-stress, that is the friction caused by the different horizontal velocities of the atmospheric winds and the ocean currents near the ocean surface. A theory of how kinetic energy is injected by the large-scale windstress into the basin-scale geostrophic ocean circulation is given in Roquet et al. (2011). At smaller scales the energy injection is, so far, less studied and understood. Furthermore, the ocean currents were commonly not taken into account when the wind-stress field was calculated, because the ocean surface currents velocities are small as compared to atmospheric winds over much of the ocean. Calculating the wind-stress using the difference between the ocean currents and the atmospheric wind, rather than the wind only, was found to lead to a twenty percent reduction of energy input in the large scale doublegyre circulation (Duhaut and Straub 2006).

As the resolution of (satellite) observations becomes ever finer in space and shorter in time, air-sea interactions at smaller scales are found to leave their imprint in the atmosphere and ocean dynamics (Chelton et al. (2004)) leading to persistent small-scale structures in the atmospheric winds. The air-sea heat flux influencing the marine atmospheric boundary layer is found in Chelton et al. (2004) to be the dominant source of the small-scale structures in the atmospheric winds. In the present work the influence of momentum transfer due to ocean dynamics is considered.

The importance of these small scale interactions for the atmosphere and ocean dynamics is confirmed using numerical simulations in idealised configurations (Hogg et al. 2009) and when applied to regional configurations (Seo et al. 2008). As the resolution of regional and global ocean models becomes ever finer in space and shorter in time, they become increasingly sensitive to more accurate and finer-resolution higher-frequency surface-windstress forcing. The importance of realistic amplitude, fine resolution and highwavenumber, surface-wind-forcing has been demonstrated in a variety of ocean modelling and climate applications. Recent fine scale satellite observations of the sea surface show an abundance of dynamical features at the oceanic meso and sub-meso scale. The explanation of the origin, turbulent dynamics and 
fate of these structures represents a formidable problem of geophysical fluid dynamics.

In the present work we exclusively focus on the exchange of momentum to evaluate its potential as a source of small scales structures in the ocean and the atmosphere. The most conspicuous feature of the atmosphere-ocean system are the different densities of the two phases, leading to a large difference in the inertia of the atmosphere and the ocean layer. At large times the atmosphere is influenced by the persistent ocean dynamics, while the short time fluctuations appear to be independent of the ocean. On the other side, short time fluctuations of the atmospheric winds have only a negligible influence on the ocean dynamics, which however responds to a persistent atmospheric forcing. This is discussed in detail by Esau (2014) for the case of the heat exchange, the case of the momentum exchange is considered here. As noted in Esau (2014) the difference in density leads in numerical models to imposing different types of boundary conditions to the atmosphere, the value is imposed (Dirichlet boundary conditions) and the ocean, the flux is imposed (von Neumann boundary conditions). This is not only the case for the heat flux (as discussed in Esau (2014)) but the same is true for the flux of inertia.

Estimates of energy fluxes are usually based on coarse-grained variables, that is variables averaged in time and space. Variables from (satellite) observations and numerical models are always coarse-grained representations of the real dynamics. When energy fluxes are estimated based on coarse-grained variables, it is often not clear how good the estimate is, or even if the true value is over- or under-estimated. The energy input and cycle is however key to understanding the dynamics of the atmosphere, ocean and climate system. The fluxes at smaller scales contribute to the turbulent mixing of substances and momentum, in the atmosphere and the ocean, without which we can not fully understand the respective dynamics. Furthermore, in the two-dimensional turbulent dynamics of the atmosphere and the ocean, energy cascades from smaller to larger scales (see e.g. Vallis (2006)), emphasising the importance of energy exchanges at small scales for the large scale dynamics.

In the here presented study, two superposed one-layer fine-resolution shallowwater models are used. The upper layer represents the atmosphere and the lower layer the ocean. Their only interaction is through a frictional force at the interface, which is parameterised by the prominent quadratic drag law. The characteristic horizontal length scales in the atmosphere and the ocean are the respective first baroclinic Rossby radii of deformation. The layer thicknesses of the atmosphere and the ocean in our model are chosen, so that the (barotropic) Rossby radii of deformation correspond to the respective length scales. The first baroclinic atmospheric Rossby radius of deformation (a few hundreds of kilometres) is usually referred to as the synoptic scale and in oceanography the first baroclinic oceanic Rossby radius of deformation (a few tenths of kilometres) is referred to as the mesoscale. All structures with a horizontal extension smaller than the synoptic scale will be called small scale, for convenience. Synoptic structures in the atmosphere rarely take more than 
a few days until they have moved away from a specific location, while the corresponding time scale for mesoscale eddies in the ocean is of a few weeks.

A fully coupled 3D-atmosphere and ocean model was used by Esau (2014) to consider heat fluxes and the induced turbulent convective dynamics in the mixed layers at the meso and sub-meso scales. He, however, considered a stressfree interface, that is without transfer of momentum. We consider momentum transfer, without including buoyancy or buoyancy fluxes. The two studies are complementary and the combination of the two effects is left for future research.

In the present work we focus on the local exchange of momentum between the ocean and the atmosphere at the synoptic, and the mesoscale. The forcing has no large scale component. The situation is very different from basin scale forcing, dynamics and flow. A basin wide forcing and circulation can create strong boundary currents which can separate from the boundary and penetrate into the interior of the domain, as is the case for the double gyre circulation (Duhaut and Straub 2006; Hogg et al. 2009), leading to strong ocean currents. To the best of our knowledge the local exchange of momentum, only, between a turbulent atmosphere and ocean, at the small scale has never been considered before.

In the present work, the qualitative dynamics of air sea-interaction is considered and the existence of two different regimes is exposed. We further assemble quantitative results on the energy fluxes and vorticity correlations between the atmosphere and the ocean for different values of the atmospheric friction coefficient.

Exchanges of heat and chemical substances are of paramount importance for the dynamics of the atmosphere, the ocean and the climate of our planet but they are not considered here as we exclusively focus on the exchange of momentum. As models of the atmosphere, the ocean and the climate system become ever more refined, further processes have to be explored and their possible impact on the dynamics has to be evaluated. It is therefore important to investigate and quantify the exchange of momentum between the synoptic and the mesoscale, the scales where the atmospheric and oceanic speeds are high.

The two superposed shallow water models and their coupling are introduced in the next section. Experiments are performed for different values of the drag coefficient given in Sect. 3, where the results concerning the energy transfer and the correlation between the vorticity dynamics in the ocean and the atmosphere are presented. The results are discussed in Sect. 4 and conclusion presented in Sect. 5. 


\section{Shallow Water Model}

\subsection{Physical model}

The model consists of two superposed homogeneous fluid layers, a shallow layer for the lower atmosphere above an ocean surface layer. The average thicknesses are respectively $H^{a}=1000 \mathrm{~m}$ and $H^{o}=200 \mathrm{~m}$. The actual layer thicknesses $h^{a}(x, y, t), h^{o}(x, y, t)$ vary over time and space. The ocean surface layer superposes a motionless layer of higher density and of infinite depth. Similarly a motionless layer of air of a lesser density superposes the shallow atmosphere layer. Layers have an average density of $\rho^{a}=1 \mathrm{~kg} \mathrm{~m}^{-3}$ and $\rho^{o}=1000 \mathrm{~kg} \mathrm{~m}^{-3}$. The fluid motion considered extends over a period of many days and so, the model must take into account the Earth's rotation. Using the f-plane approximation we set the Coriolis parameter $f=10^{-4} \mathrm{~s}^{-1}$, a typical value at mid-latitudes.

\subsection{Mathematical model}

This physical model can be described by two coupled reduced-gravity shallow water equations:

$$
\begin{gathered}
\partial_{t} u^{k}+u^{k} \partial_{x} u^{k}+v^{k} \partial_{y} u^{k}-f v^{k}+g^{k} \partial_{x} h^{k}=\nu^{k} \nabla^{2} u^{k}+F_{x}^{k} \\
\partial_{t} v^{k}+u^{k} \partial_{x} v^{k}+v^{k} \partial_{y} v^{k}+f u^{k}+g^{k} \partial_{y} h^{k}=\nu^{k} \nabla^{2} v^{k}+F_{y}^{k} \\
\partial_{t} h^{k}+\partial_{x}\left[h^{k} u^{k}\right]+\partial_{y}\left[h^{k} v^{k}\right]=\tilde{F}_{h}^{k}
\end{gathered}
$$

where $k=a, o$ stands for the atmosphere and the ocean, respectively. The parameters $g^{a}$ and $g^{o}$ are the reduced gravity of the atmosphere and of the ocean (i.e., the gravitational acceleration multiplied by the fractional density difference between the two layers). They are respectively set to $0.8 \mathrm{~m} \mathrm{~s}^{-2}$ and $2.10^{-2} \mathrm{~m} \mathrm{~s}^{-2}$. The restoring force $\tilde{F}_{h}^{k}$ in the atmosphere and ocean acts on the layer-thickness.

The typical horizontal scale is the Rossby radius of deformation $R d^{k}=$ $\sqrt{g^{k} H^{k}} / f$. It is about one order of magnitude smaller in the ocean where $R d^{o}=20 \mathrm{~km}$ than in the atmosphere where $R d^{a} \simeq 283 \mathrm{~km}$. The domain size is $L_{x}=L_{y}=4000 \mathrm{~km}$ and there are periodic boundary conditions in both horizontal directions. In the absence of forcing and friction the potential vorticity $(\mathrm{PV})$ :

$$
q^{k}=\frac{\zeta^{k}+f}{h^{k}} \text { with } \zeta^{k}=\partial_{x} v^{k}-\partial_{y} u^{k}
$$

is conserved by the flow.

The initial oceanic height variation is defined by the sum of the $2 \pi$ periodic cosinus in the $x$ - and $y$ - direction.

$$
\eta_{o}^{a}(x, y)=50 \mathrm{~m} \times\left(\cos \left(\frac{2 \pi(\mathrm{x}-7 / 24)}{\mathrm{L}_{\mathrm{x}}}\right)+\cos \left(\frac{2 \pi(\mathrm{y}-7 / 24)}{\mathrm{L}_{\mathrm{y}}}\right)\right)
$$


The initial velocity field is calculated using the geostrophic equilibrium.

The initial atmospheric height variation is defined by periodic alternating cyclones and anticyclones in the $x$ and $y$ direction, with a period of $1000 \mathrm{~km}$.

$$
\begin{aligned}
\eta_{o}^{a}(x, y)=350 \mathrm{~m} \times & {\left[\cos \left(8 \pi x / L_{x}\right)+\cos \left(16 \pi x / L_{x}\right)+\cos \left(24 \pi x / L_{x}\right)\right.} \\
& \left.+\cos \left(8 \pi y / L_{y}\right)\right)+\cos \left(16 \pi y / L_{y}\right)+\cos \left(24 \pi y / L_{y}\right) \\
& \left.+\cos \left(8 \pi(x+y) / L_{x}\right)+\cos \left(16 \pi(x+y) / L_{x}\right)\right]
\end{aligned}
$$

The initial velocity field is calculated using the geostrophic equilibrium. So four cyclonic and anti-cyclonic alternating structures are imposed on the $x$ and on the $y$ direction, leading to 16 identical tiles in the forcing field.

A restoring acts to force the average of the atmospheric layer thickness projected on the forcing profile (Eq.6) towards its initial value. To this end, the projection is compared to its initial value and a multiple of the initial profile is added or subtracted to restore towards the initial amplitude of the projected mode. Such kind of restoring directly affects only the forced mode without directly influencing the other modes which can evolve more freely. The restoring time is two days. The variation of layer thickness in the ocean layer is locally and linearly damped to zero with a long damping time of 1000 days, in order to not disturb the air-sea interaction.

When parameterizing the effect of small-scale turbulent friction at a solid boundary a quadratic drag law is used. Such a drag law dates back to the work of Prandtl and Schlichting (1934) and Schlichting and Gertsen (2000) and has been extensively studied since then (see Schlichting Gertsen (2000)). All these investigations essentially confirm its robustness and its applicability to all fields of fluid dynamics. When the motion of the atmosphere and the ocean are considered a large volume of research is dedicated to the determination of the drag coefficient over various surfaces (Stull 1988). When the sea surface is considered the drag coefficient depends on the sea-state, which itself is a function of various parameters (see e.g. Högström et al. 2013). The robustness of the law itself seems above any doubt.

The two layers are only linked by frictional forces at the interface, parameterised by a quadratic drag law. The frictional acceleration between the two layers (see eqs. 1 and 2 ) is defined by:

$$
\left(\begin{array}{c}
F_{x}^{k} \\
F_{y}^{k}
\end{array}\right)= \pm \frac{1}{\rho^{k} h^{k}}\left(\begin{array}{c}
f_{x}^{k} \\
f_{y}^{k}
\end{array}\right),
$$

where $f_{x}$ and $f_{y}$ are the surface forces varying in $x, y$ and time. They are calculated using the velocity difference between wind and ocean current,

$$
\left(\begin{array}{c}
f_{x}^{o} \\
f_{y}^{o}
\end{array}\right)=\rho^{a} C_{d}|u|\left(\begin{array}{c}
u^{o}-u^{a} \\
v^{o}-v^{a}
\end{array}\right),
$$

with the square of the relative speed $u^{2}=\left(u^{o}-u^{a}\right)^{2}+\left(v^{o}-v^{a}\right)^{2}$. The shear applied to the atmosphere is the opposite to the shear applied to the ocean.

The drag coefficient $C_{d}$ is constant within each experiment. 
2.3 Numerical model

The ocean and the atmosphere basins are represented by a rectangle, of $L_{x} \times$ $L_{y}$. Periodic boundary conditions are used in both horizontal directions. The numerical grid is regular and contains $n_{x} \times n_{y}$ points. Fine spacial resolutions $\Delta x=L_{x} / n_{x}=\Delta y=L_{y} / n_{y}$ are employed to well resolved the horizontal scales. We choose $n_{x}=n_{y}=2048$ and $L_{x}=L_{y}=4000 \mathrm{~km}$ for the $2 \mathrm{D}$ shallow water model. The horizontal components of the velocity $u^{k}, v^{k}$, and height variations $\eta^{k}$ are calculated on each grid point. The eddy viscosity of the layers are $\nu^{a}=500 \mathrm{~m}^{2} \mathrm{~s}^{-1}$ and $\nu^{o}=0.1 \mathrm{~m}^{2} \mathrm{~s}^{-1}$, which are constants in space and time.

A second order centred finite difference method is used for the space discretization and a second-order Runge-Kutta scheme is used for the time discretization. The timestep is constraint by the Courant-Friedrichs-Lewy condition (see Courant et al, 1928). It states that the timestep must be less than the time for the wave to travel to adjacent grid points. As atmospheric waves are ten times faster than oceanic waves, it is the Courant-Friedrichs-Lewy condition for the atmosphere that sets the minimum timestep $\Delta t=15 \mathrm{~s}$ to well resolve the temporal evolution of the atmospheric dynamics.

The model has been already employed in Moulin and Wirth (2014) at lower resolution in a non-parallelised version.

\section{Results}

\subsection{Experiments Performed}

We present results from four numerical experiments which only vary by the drag coefficient used. The drag coefficient $C_{d}$ is constant for each experiment. In the four simulations discussed here, the drag coefficient is $C_{d}=1 \times 10^{-4}$, $2 \times 10^{-4}, 4 \times 10^{-4}$ and $8 \times 10^{-4}$. The values used are within the typical values for ocean dynamics.

The drag coefficient depends on a variety of parameters as the ocean state and the density stratification of the atmosphere. When the motion of the atmosphere and the ocean are considered a large volume of research is dedicated to the determination of the drag coefficient over various surfaces (e.g. Stull 1988; Högström et al. 2013) and atmospheric conditions. In the shallow water equations the velocity vertically averaged over the whole layer thickness, is represented. This explains, why these geostrophic drag coefficients are lower than the drag coefficients based on velocities at $10 \mathrm{~m}$ above the ocean surface (e.g., Stull (1988)). According to Stull (1988) the drag coefficient for a reference level of about $500 \mathrm{~m}$ is typically between $1 \times 10^{-4}$ for stable atmosphere and $8 \times 10^{-4}$ for a neutral atmosphere.

Integrations are performed over 2700 days. The last 1000 days are considered for temporal averages. This ensures that the calculations have converged close to a statistically stationary state. 
3.2 Qualitative Description

In all the four experiments a nonlinear dynamics is observed in the atmosphere and the ocean. The speeds and the absolute vorticity values of the ocean increase with the drag coefficient, the opposite is true for the atmosphere, as expected. The structures in the ocean are smaller than those in the atmosphere, showing that both developed their own dynamics. The difference in size is readily explained by the different Rossby radii of deformation. For all values of the drag coefficient, a correspondence between the vorticity in the atmosphere and the ocean is observed, showing that the dynamics in the atmosphere and the ocean is co-organised.

For the smaller values of the drag coefficient the co-organisation is only visible in the averages (see Fig. 1, lower panels), as the atmospheric perturbations are strong and evolve on a much smaller time scale than the ocean. The shortterm instantaneous perturbations in the atmosphere are almost transparent to the ocean, which only adapts to a time-integrated atmospheric dynamics. The atmospheric dynamics appears independent of the ocean dynamics and the external forcing on the atmosphere, when instantaneous values are considered (see Fig. 1 upper panels). In the instantaneous vorticity field of the experiment with the lowest drag coefficient, the 16 cells of the space periodic forcing are visible in the ocean but not in the atmosphere, although the forcing is applied to the atmosphere and not to the ocean, which experiences it only through the atmospheric dynamics. When long-time averages are considered the forcing is also the dominant signal in the atmosphere. This shows that the ocean adapts to a time-averaged atmosphere. For the smaller drag coefficients there are only a few coherent structures in the ocean. The velocities are too small for the non-linear terms to become important, and their dynamics is hindered by the strong atmospheric turbulence representing a high frequency forcing on the ocean dynamics. Inspection of movies show that at times, the atmospheric eddies are attracted by like-sign ocean vorticity structures and they superpose during few days. This injects further vorticity in the ocean and leads to stronger eddies during a laps of time. These transient structures in the ocean disappear after the atmospheric eddy has moved and the highfrequency forcing of to the atmosphere disintegrates the structures.

For the largest value of the drag coefficient the situation is qualitatively different. A variety of coherent structures dominate the vorticity filed in the ocean and the atmosphere and they appear co-located (see Fig. 2). The temporal vorticity anomaly in the atmosphere is strongly damped (see Fig. 2 upper-left panel) and the atmosphere and ocean dynamics is in a quenched disordered state. That is, a spatial disorder with a very slow evolution in time, in both the atmosphere and the ocean is observed. Such type of dynamics is found and extensively studied in spin-glasses but has so far not been mentioned in the context of air-sea interaction, to the best of our knowledge. The atmosphere is in a quasi-stationary state. This allows the ocean to develop larger eddies (Fig. 2). After a few years, the ocean and the atmosphere have a similar dynamics at large scale with many co-located features. As we see in 

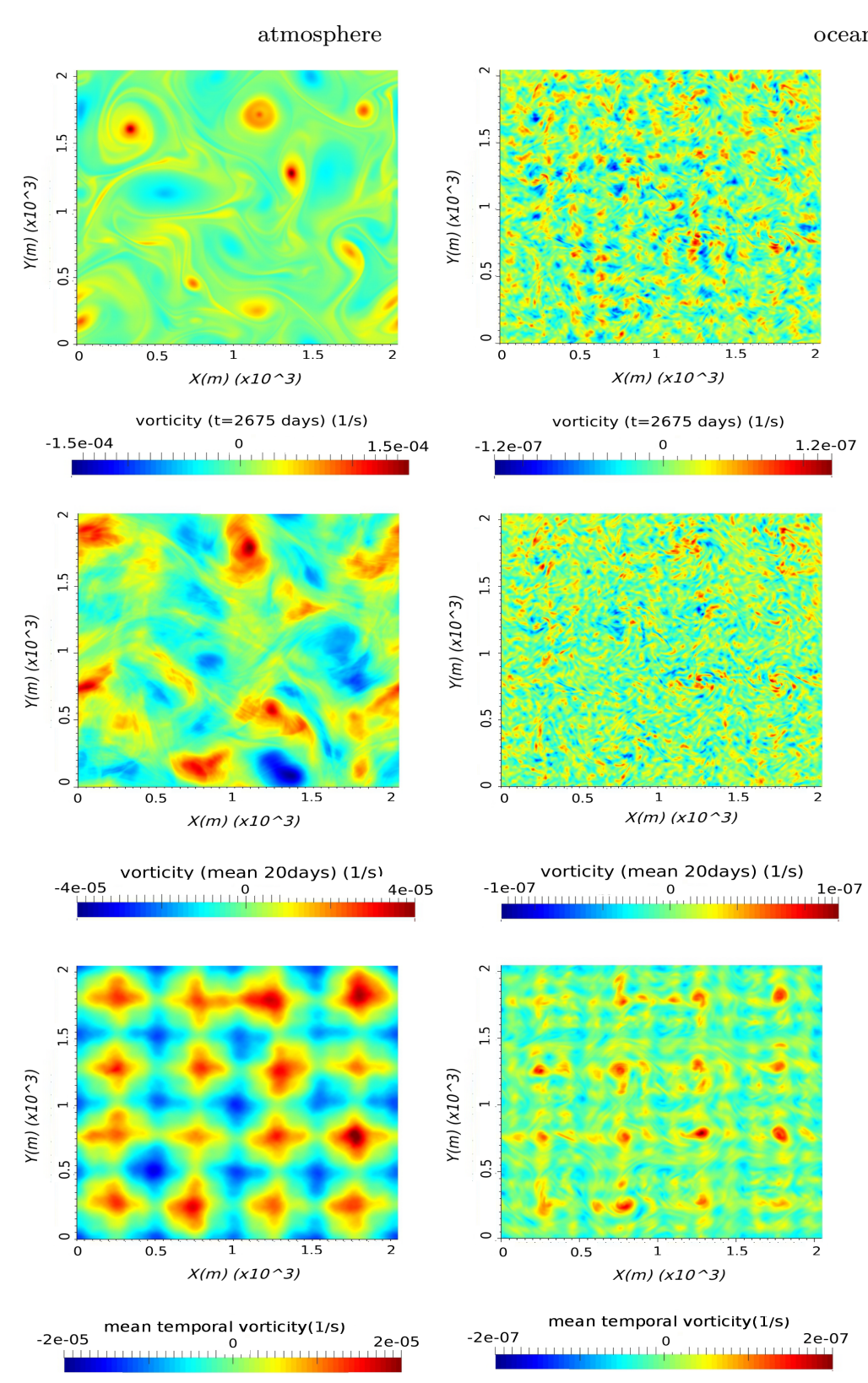

Fig. 1 For $C_{d}=1 \times 10^{-4}$. Map of the average vorticity, averaged over 1000 days, starting from day 1700 (bottom). Map of the vorticity anomaly, averaged over 20 days, starting from day 2680 , with respect to the 1000 days average (middle). Map of the instantaneous vorticity anomaly at day 2675 , with respect to the 1000 days average (top). 

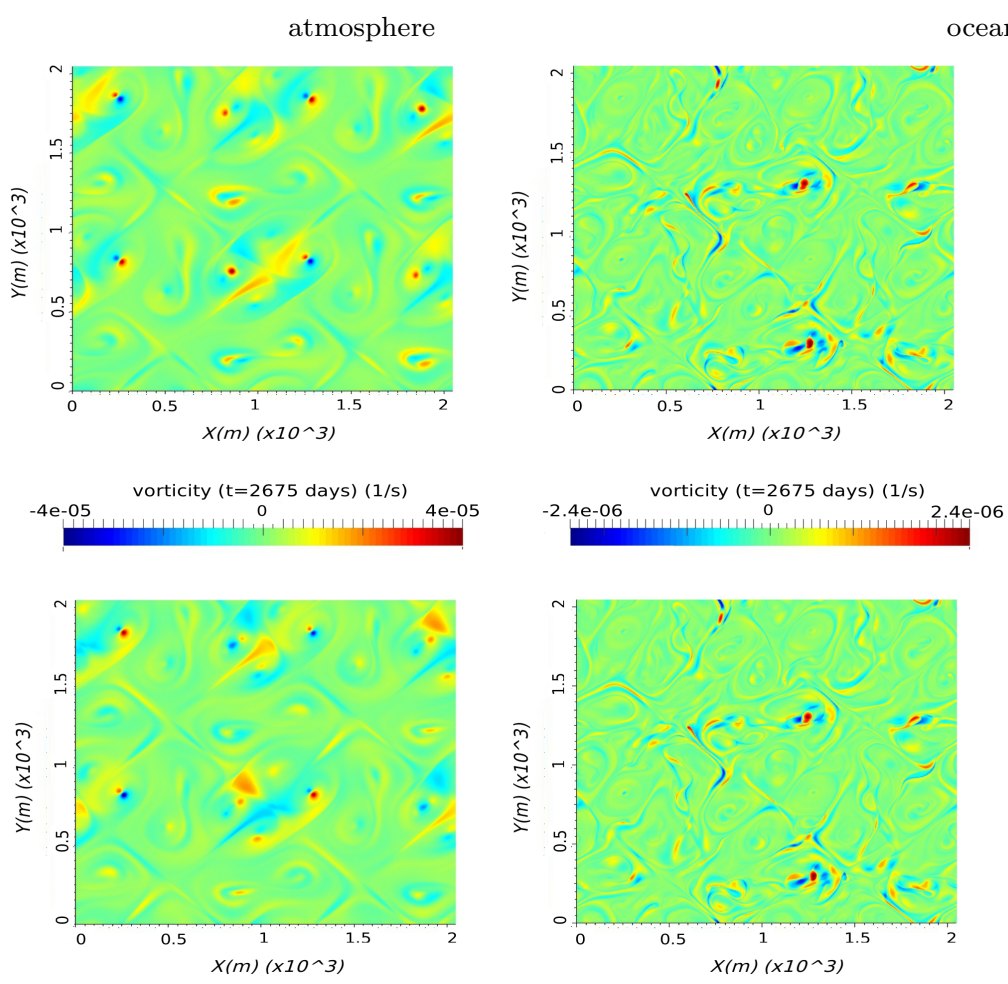

vorticity (mean 20days) (1/s)
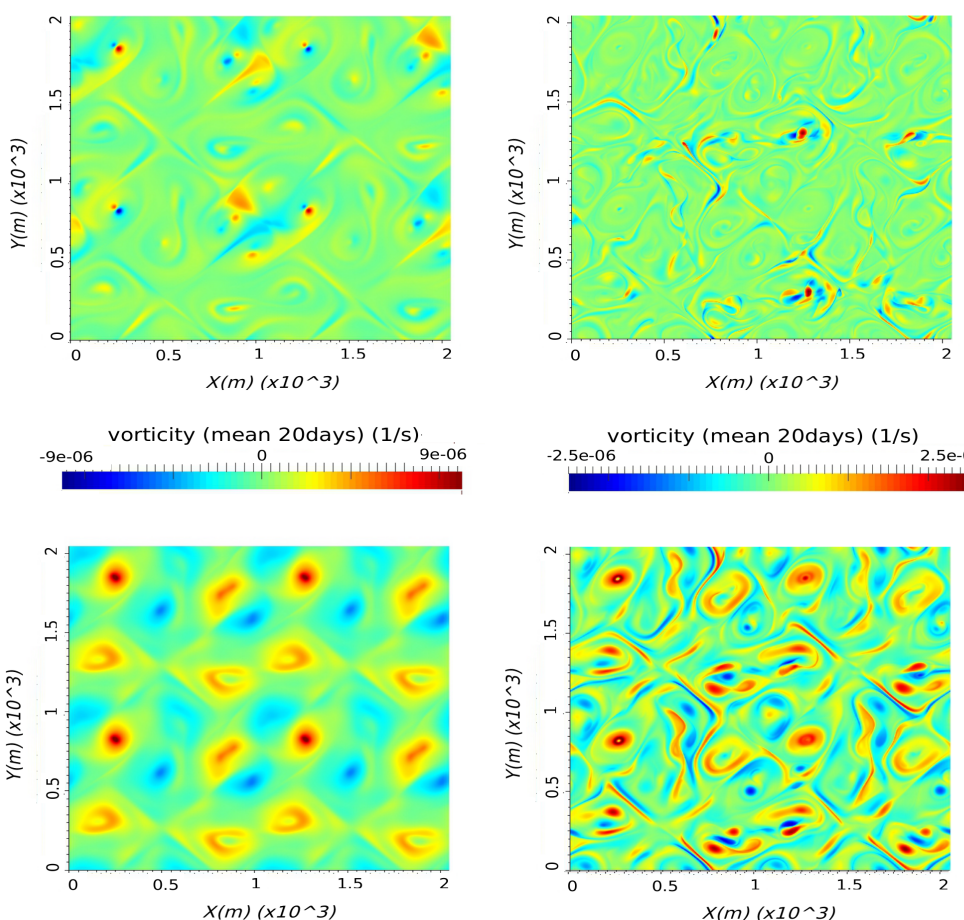

vorticity (mean 20days) (1/s)
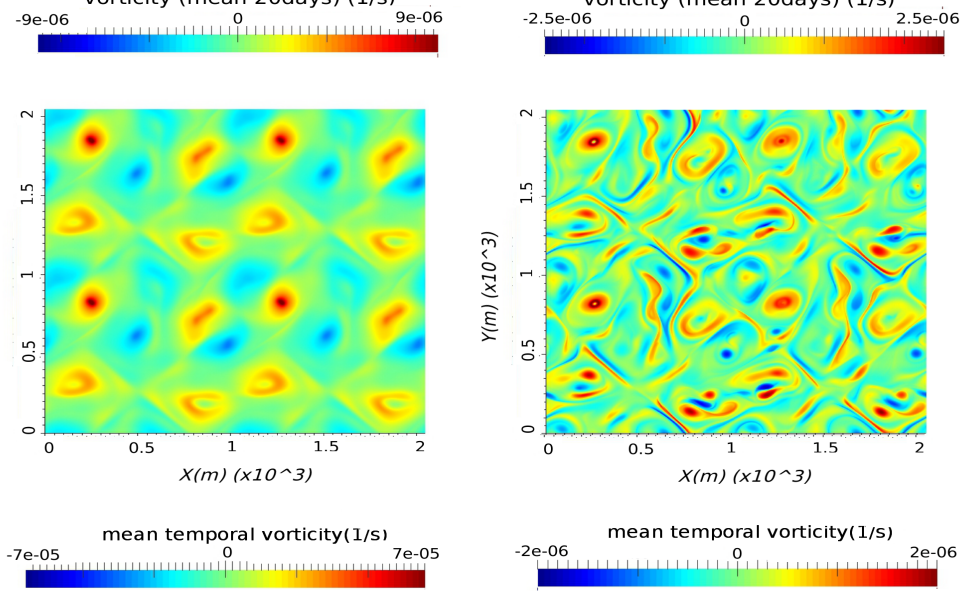

Fig. 2 For $C_{d}=8 \times 10^{-4}$. Map of the average vorticity, averaged over 1000 days, starting from day 1700 (bottom). Map of the vorticity anomaly, averaged over 20 days, starting from day 2680 , with respect to the 1000 days average (middle). Map of the instantaneous vorticity anomaly at day 2675 , with respect to the 1000 days average (top). 
Fig. 2, the mean vorticity, averaged over 1000 days, is strong as compared to the temporal vorticity anomalies, in both the atmosphere and the ocean. In the atmosphere the vorticity anomaly shows the formation of dipole structures, where there is a strong mean vorticity. They are the signature of the displacement of vortices. Many of the atmospheric eddies have a ring-structure, due to opposite vorticity in the ocean at the centre of the atmospheric eddy. When no opposite vorticity is present in the ocean, the atmospheric eddies have stronger vorticity.

\subsection{Kinetic energy transfer}

The density differences between the air and the water masses leads to a large discrepancy between the speeds in the ocean and the atmosphere as shown in Table 1. When increasing the drag coefficient eight fold, the atmospheric r.m.s velocity decreases by about $40 \%$, while the oceanic r.m.s velocity increases almost an order of magnitude. This is also visible in the ratio between the kinetic energy in the ocean and the atmosphere, which is over 200 times higher with the highest drag coefficient as compared to the lowest (Table 1).

The mechanical power input per unit surface area to the ocean is the vector-product of the wind-stress shear $\tau$ and the ocean velocity $\mathbf{u}_{\mathbf{o}}$, that is: $P=\tau \cdot \mathbf{u}_{\mathbf{o}}$ (e.g., Duhaut and Straub 2006). The power input using the correct representation of the shear force, is based on the velocity difference between the atmospheric and the oceanic velocity $\tau_{\mathbf{c}}=\rho_{a} C_{d}\left|\mathbf{u}_{\mathbf{a}}-\mathbf{u}_{\mathbf{o}}\right|\left(\mathbf{u}_{\mathbf{a}}-\mathbf{u}_{\mathbf{o}}\right)$. The approximation where the effect of the ocean current is neglected when calculating the shear $\tau_{\mathbf{a p p}}=\rho_{a} C_{d}\left|\mathbf{u}_{\mathbf{a}}\right| \mathbf{u}_{\mathbf{a}}$ is often used. It is justified by the fact, that the ocean currents are feeble as compared to the wind speeds $\left(u_{o} \ll u_{a}\right)$. Duhaut and Straub (2006) revealed an overestimation of about $20 \%$ of the power input to the ocean, in a double gyre configuration with a prescribed time-independent wind velocity, when the approximation is used. It can be shown that the correct values of the power input $P_{c}$ are always smaller than the approximated values $P_{a p p}$. In our results, there is almost no difference between $P_{c}$ and $P_{a p p}$ for the smaller drag coefficients, because the ocean speeds are very small as compared to the atmospheric speed. For the highest drag coefficient the energy input estimated with the approximation overestimates the power input by $5 \%$. The difference is accentuated for larger drag coefficients as higher velocities are induced in the ocean, and the ocean velocities are more aligned with the atmospheric velocities (Fig. 2). The fact that on average the difference is low, does not mean, that locally it is not important, in areas where oceanic currents are strong and aligned to the atmospheric winds.

On average, the atmosphere loses energy over more than 95 percent of its surface area for all drag coefficient (Table 1). The percentage increases with a decreasing drag coefficients, attaining $99 \%$ for the smallest drag coefficient. This shows, that for the atmospheric kinetic energy, the ocean has a passive role. The loss of kinetic energy is of the same order of magnitude for all drag coefficients. The main part of the kinetic energy lost by the atmosphere in 
Table 1 Averaged quantities for the four values of the drag coefficient. Mean power lost/gained by the atmosphere/ocean for the consistent calculation $\left({\overline{\mathrm{Pa}\left(u_{a}\right)}}^{t},{\overline{\mathrm{Po}\left(u_{a}, u_{o}\right)}}^{t}\right.$ ) and power lost/gained based on the mean atmospheric/oceanic velocity $\left(\mathrm{Pa}\left({\overline{u_{a}}}^{t}\right)\right.$, $\left.\operatorname{Po}\left({\overline{u_{a}}}^{t},{\overline{u_{o}}}^{t}\right)\right)$.

\begin{tabular}{|l|c|c|c|c|}
\hline$C_{d}$ & $1 \times 10^{-4}$ & $2 \times 10^{-4}$ & $4 \times 10^{-4}$ & $8 \times 10^{-4}$ \\
\hline$\sqrt{u_{a}^{2}}\left(\mathrm{~m} \mathrm{~s}^{-1}\right)$ & 3.3 & 2.9 & 2.5 & 2.0 \\
\hline$\sqrt{u_{o}^{2}}\left(\mathrm{~m} \mathrm{~s}^{-1}\right)$ & $3.4 \times 10^{-3}$ & $6.4 \times 10^{-3}$ & $1.3 \times 10^{-2}$ & $3.1 \times 10^{-2}$ \\
\hline$E_{a} / E_{o}$ & $4.6 \times 10^{3}$ & $1.1 \times 10^{3}$ & $2.2 \times 10^{2}$ & 22 \\
\hline $\begin{array}{l}\text { surface fraction where } \\
\text { the atmosph. losses en- } \\
\text { ergy(\%) }\end{array}$ & 99 & 98 & 97 & 95.5 \\
\hline $\begin{array}{l}\text { surface fraction where } \\
\text { the ocean gains en- } \\
\text { ergy }(\%)\end{array}$ & 58 & 70 & 82 & 77 \\
\hline$\left.P a\left({\overline{u_{a}}}^{t}\right)(\mathrm{W} \mathrm{m})^{-2}\right)$ & $-3.6 \times 10^{-3}$ & $-4.9 \times 10^{-3}$ & $-6.3 \times 10^{-3}$ & $-6.4 \times 10^{-3}$ \\
\left.\hline${\overline{P a}\left(u_{a}\right)}^{t}(\mathrm{~W} \mathrm{~m})^{-2}\right)$ & $-4.6 \times 10^{-3}$ & $-6.1 \times 10^{-3}$ & $-7.5 \times 10^{-3}$ & $-7.0 \times 10^{-3}$ \\
\hline$\left.P o\left(\bar{u}_{a}^{t},{\overline{u_{o}}}^{t}\right)(\mathrm{W} \mathrm{m})^{-2}\right)$ & $2.6 \times 10^{-6}$ & $7.9 \times 10^{-6}$ & $2.4 \times 10^{-5}$ & $7.0 \times 10^{-5}$ \\
\left.\hline${\overline{P o}\left(u_{a}, u_{o}\right)}^{t}(\mathrm{~W} \mathrm{~m})^{-2}\right)$ & $8.7 \times 10^{-7}$ & $5.2 \times 10^{-6}$ & $2.1 \times 10^{-5}$ & $8.3 \times 10^{-5}$ \\
\hline $\begin{array}{l}\text { Taylor scale in the at- } \\
\text { mosphere }(\mathrm{km})\end{array}$ & 127 & 121 & 112 & 116 \\
\hline $\begin{array}{l}\text { Taylor scale in the } \\
\text { ocean }(\mathrm{km})\end{array}$ & 68 & 74 & 83 & 45 \\
\hline
\end{tabular}

the air-sea interaction is dissipated. Indeed, only 3 percent is transferred to the ocean for the highest drag coefficient, it decreases to 0.06 percent for the smallest drag coefficient. This can again be explained by the lower speeds in the ocean as compared to the atmosphere and by the non-alignment between the local velocity vectors.

The ocean gains energy from the atmosphere, whenever the absolute value of the angle between the ocean current and the shear force exerted by the atmospheric wind is smaller than $90^{\circ}$. Note that the direction of the shear force is aligned with the difference of the atmospheric wind vector and the ocean current vector. For the smaller drag coefficient the ocean gains energy from the atmosphere over $58 \%$ of its surface area, against $77 \%$ for the highest drag coefficient (Table 1). This is explained by the stronger coupling between the atmosphere and ocean dynamics at higher drag coefficients.

Figure 3 shows histograms of the wind speeds (left) and the ocean speeds (right). Superposed is the percentage of energy lost by the atmosphere for the corresponding speed range. The percentage of the energy gained by the ocean for the corresponding speed range is shown by the blue curve. The thick vertical line is the mean velocity. Due to the cubic law for the energy loss, the atmosphere loses its power at its high atmospheric speeds (Fig. 3). A comparison of the real energy loss to its approximation calculated with the mean velocity $\left(\overline{P a}=\rho_{a} C_{d}{\overline{u_{a}}}^{3}\right)$, shows that it underestimates the energy loss by almost a factor of one-and-a-half for the lowest drag coefficients and provides a good estimate for the largest (Table 1). 
The power input to the ocean is principally at the same atmospheric ve4 locities at which the atmosphere loses energy, as is seen in Fig. 3, where the red full line and the blue dashed line are similar in the figures corresponding to the atmospheric speeds (left column).

The power input to the ocean (Fig. 3, right column) shows a strong dependence on the drag coefficient $C_{d}$. For the lowest $C_{d}$ value, $14 \%$ of the fastest ocean speeds contribute half of the power input. For the largest $C_{d}$, this is achieved by only the fastest $3 \%$ of ocean speeds. An explanation of this is an increased correlation between oceanic and atmospheric velocities for higher drag coefficients (see below). This result questions the significance of mean wind and current speeds when the power input is estimated. Indeed, as a rule of thump, the power input from the atmosphere to the ocean is often estimated based on the mean wind and ocean speeds. In this estimation not only the correlation of the local wind and ocean speeds are neglected, but also the angle between the corresponding velocity vectors. The estimated value is compared to the exact value in Table 1 . For the highest drag coefficient the estimated value is $16 \%$ lower than the true value, while it is 3 times higher for the smallest drag coefficient (Table 1). The higher value of the estimate is explained by the fact that it neglects the non-alignment between atmospheric wind and the ocean currents. The non-alignment is highest for the lower values of the drag coefficient. The lower value of the estimate is explained by the correlation of the magnitudes of the atmospheric and oceanic speeds.

Performing a log-log plot of the power-input to the ocean (averaged in space and time) as a function of the drag coefficient (Fig. 4) we see, that it is close to a quadratic law. Note, that the drag coefficient has a stronger than linear influence on the power input to the ocean as a higher drag coefficient increases the shear force and the velocity in the ocean and the power-input is the (vector) product of the two. A higher correlation between atmosphere and ocean velocities for higher drag coefficients further increase the power input.

\subsection{Vorticity correlation between the atmosphere and the ocean}

The normalised bi-variate probability density function for the atmospheric and the oceanic vorticity is used, to study the correlation between the dynamics in the ocean and the atmosphere. It is obtained by first subtracting the mean-value and then normalising the atmospheric and oceanic vorticity by their respective standard deviation, before considering the correlations. For an instantaneous snapshot after 7 years, and the smallest drag coefficient, the isolines of the probability density function and the overlaid correlation ellipse appear almost circular (Fig. 5). Indeed the ratio between the two principal correlations is 1.48 (Table 2), close to 1 which is the value for a perfect decorrelation. This shows that there is only a feeble correlation between the atmospheric and oceanic vorticities. The correlation increases for the drag coefficients $C_{d}=2 \times 10^{-4}$ and $C_{d}=4 \times 10^{-4}$ before slightly dropping to 2.24 for $C_{d}=8 \times 10^{-4}$. For the vorticities averaged over 1000 days the cross-correlation 
Table 2 Variance and spatial average of the vorticity in the ocean and in the atmosphere for an instantaneous snapshot (after 2675 days) above and for a temporal average over 1000 days (below).

\begin{tabular}{|l|c|c|c|c|}
\hline$C_{d}$ & $1 \times 10^{-4}$ & $2 \times 10^{-4}$ & $4 \times 10^{-4}$ & $8 \times 10^{-4}$ \\
\begin{tabular}{|l|c|c|c|c|}
\hline \multicolumn{3}{|c|}{ Instantaneous } \\
variance of atmo- \\
spheric vorticity $\left(\mathrm{s}^{-2}\right)$
\end{tabular} & $3.28 \times 10^{-10}$ & $3.35 \times 10^{-10}$ & $2.77 \times 10^{-10}$ & $1.71 \times 10^{-10}$ \\
\hline $\begin{array}{l}\text { variance of oceanic vor- } \\
\text { ticity (s }\end{array}$ & $1.35 \times 10^{-15}$ & $4.43 \times 10^{-15}$ & $1.49 \times 10^{-14}$ & $2.37 \times 10^{-13}$ \\
\hline $\begin{array}{l}\text { ratio between the big } \\
\text { and the small half axes } \\
\text { of the ellipse }\end{array}$ & 1.48 & 1.74 & 2.28 & 2.24 \\
\hline slope of principal axis & $2.03 \times 10^{-3}$ & $3.64 \times 10^{-3}$ & $7.33 \times 10^{-3}$ & $3.73 \times 10^{-2}$ \\
\hline
\end{tabular}

\begin{tabular}{|l|c|c|c|c|}
\hline $\begin{array}{l}\text { variance of atmo- } \\
\text { spheric vorticity }\left(\mathrm{s}^{-2}\right)\end{array}$ & $3.60 \times 10^{-11}$ & $5.85 \times 10^{-11}$ & $9.90 \times 10^{-11}$ & $1.60 \times 10^{-10}$ \\
\hline $\begin{array}{l}\text { variance of oceanic vor- } \\
\text { ticity }\left(\mathrm{s}^{-2}\right)\end{array}$ & $9.58 \times 10^{-16}$ & $3.25 \times 10^{-15}$ & $1.18 \times 10^{-14}$ & $1.97 \times 10^{-13}$ \\
\hline $\begin{array}{l}\text { ratio between the big } \\
\text { and the small half axes } \\
\text { of the ellipse }\end{array}$ & 3.66 & 4.91 & 5.45 & 2.57 \\
\hline slope of principal axis & $5.17 \times 10^{-3}$ & $7.45 \times 10^{-3}$ & $1.09 \times 10^{-2}$ & $3.52 \times 10^{-2}$ \\
\hline
\end{tabular}

is higher than the instantaneous values. The ratio between the two principal correlations is more than 2 times higher than for the instantaneous snapshot (Table 2) for the lower three drag coefficients, confirming the above mentioned observation that, when the fast variations of the atmosphere are filtered out the correlation between the ocean and the atmosphere increases. For the highest drag coefficient the dynamics is qualitatively different: the time variability, in the atmosphere and the ocean, is low as the dynamics is in a quenched state (see next subsection) and the cross correlation increases only by $15 \%$ when averages, rather than instantaneous values are considered.

When the normalised bi-variate probability density function is considered the slope of the principal axis, which is the ratio of the standard deviation of the ocean and the atmosphere, is diagonal by definition (see Figs. 5 and 6 ). In the non normalised case it establishes a correspondence between the vorticity in the two media. The slope of the first principal axis is about two times stronger for the temporal mean than for the snapshot, for the three smaller drag coefficients. For the largest drag coefficient the two slopes are similar as there is almost no variation in time.

\subsection{Spatial versus Temporal Variability of the Vorticity}

In this part we study the variability in space and time of the vorticity field in the atmosphere and the ocean as a function of the drag coefficient. We need to define two different types of average: a spatial average and a temporal average. The temporal average of the vorticity has already been defined in the 
Table 3 Variability in space (varSpat) and time (vartemp) for the four drag coefficient for the ocean and the atmosphere.

\begin{tabular}{|c|c|c|c|c|}
\hline$C_{d}$ & $1 \times 10^{-4}$ & $2 \times 10^{-4}$ & $4 \times 10^{-4}$ & $8 \times 10^{-4}$ \\
\hline varTota $\left(\mathrm{s}^{-2}\right)$ & $3.48 \times 10^{-10}$ & $2.77 \times 10^{-10}$ & $1.76 \times 10^{-10}$ & $9.57 \times 10^{-11}$ \\
\hline varToto $\left(\mathrm{s}^{-2}\right)$ & $8.64 \times 10^{-16}$ & $1.69 \times 10^{-15}$ & $4.86 \times 10^{-15}$ & $2.16 \times 10^{-13}$ \\
\hline varSpata $\left(\mathrm{s}^{-2}\right)$ & $6.17 \times 10^{-12}$ & $3.89 \times 10^{-12}$ & $5.69 \times 10^{-12}$ & $8.32 \times 10^{-11}$ \\
\hline varSpato $\left(\mathrm{s}^{-2}\right)$ & $3.46 \times 10^{-16}$ & $7.65 \times 10^{-16}$ & $2.25 \times 10^{-15}$ & $1.79 \times 10^{-13}$ \\
\hline vartempa $\left(\mathrm{s}^{-2}\right)$ & $3.43 \times 10^{-10}$ & $2.73 \times 10^{-10}$ & $1.70 \times 10^{-10}$ & $1.27 \times 10^{-11}$ \\
\hline vartempo $\left(\mathrm{s}^{-2}\right)$ & $5.18 \times 10^{-16}$ & $9.30 \times 10^{-16}$ & $2.60 \times 10^{-15}$ & $3.63 \times 10^{-14}$ \\
\hline
\end{tabular}

first part. It is the average of the vorticity over 1000 days denoted by $\left\langle\zeta^{k}\right\rangle_{1000}$. Where $\mathrm{k}$ corresponds to the ocean or the atmosphere.

The forcing applied to our periodic domain repeats itself 4 times in the $x$ - and in the $y$-direction, leading to 16 identical cells. Furthermore, rotations of angles 90, 180 and 270 degrees do not change the forcing. The discrete symmetry group of the forcing has therefore 64 members. The dynamics in the atmosphere and the ocean, does not exhibit this periodicity, as it is not present in the initial conditions and as the system is chaotic. There are $4 \times 4$ identically forced squares present in the domain and we expect to find the periodicity of the forcing in the averages of dynamical quantities as there is a priory no reason to suppose that averages differ from one square to another once the initial conditions are "forgotten". That is, we suppose the system to be ergodic, meaning that ensemble averages and time averages coincide.

For the calculation of the spatial average we use the full symmetry group with 64 members. We calculate the average over all members of size $1000 \mathrm{~km} \times 1000 \mathrm{~km}$, and continue it periodically over the large domain of size $4000 \mathrm{~km} \times 4000 \mathrm{~km}$.

This discrete spatial average is denoted by $\bar{\zeta}^{64}$.

To calculate the variability we also need to define the spatial variance:

$$
\operatorname{var}(a)=\frac{1}{n_{x} n_{y}} \sum_{i, j=0}^{n_{x}, n_{y}}\left(a_{i, j}\right)^{2},
$$

where a is a matrix of size $n_{x} \times n_{y}$.

With all these terms the spatial variability can be defined by:

$$
\operatorname{Var} S^{k}=\operatorname{var}\left(\left\langle\zeta^{k}\right\rangle_{1000}-{\overline{\left\langle\zeta^{k}\right\rangle_{1000}}}^{64}\right) .
$$

The space variability reveals the difference obtained when we reproduced the experiment with a little initial perturbation.

The temporal variability represents the evolution of the variable in time, it can be defined by:

$$
\operatorname{Vart}^{k}=\left\langle\operatorname{var}\left(\zeta^{k}-\left\langle\zeta^{k}\right\rangle_{1000}\right)\right\rangle_{1000} .
$$

The total variability is written:

$$
\operatorname{VarTot}{ }^{k}=\left\langle\operatorname{var}\left(\zeta^{k}-{\overline{\left\langle\zeta^{k}\right\rangle_{1000}}}^{64}\right)\right\rangle_{1000} .
$$


It can be shown that the total variability is the sum of the spatial and the temporal variability:

$$
\operatorname{VarTot}^{k}=\operatorname{Var} S^{k}+\operatorname{Vart}^{k} .
$$

The results, for the four drag coefficient, are plotted in an histogram for the atmosphere (Fig. 7 (left)) and for the ocean (Fig. 7 (right)). In the atmosphere, the total variability decreases when the drag coefficient increases. The spatial variability is small $(\ll 3 \%)$ for the the three lower drag coefficients but skyrockets to $86 \%$ for $C_{d}=8 \times 10^{-4}$ of the total variability, showing that the dynamics changes qualitatively when the drag coefficient is increased from $C_{d}=4 \times 10^{-4}$ to $8 \times 10^{-4}$. In the ocean, the total variability is very low for the smallest three drag coefficient (order of $10^{-15} \mathrm{~s}^{-2}$ ) with again a strong dominance of the the temporal variability. The case with the strongest drag coefficient is again very different from the lower ones. It has a total variability around hundred times larger with more than three quarters due to the spatial variation. The disorder in the highest drag coefficient case is dominated by spatial disorder (quenched disorder), whereas the three other cases, the temporal disorder prevails (annealed disorder).

\section{Discussion}

From an kinetic-energy point of view the atmosphere-ocean system is driven by the injection of kinetic energy into the atmosphere. The system losses energy due to friction at the atmosphere-ocean interface (and to a lesser extent by horizontal friction, not discussed here). A small fraction of the kinetic-energy lost by the atmosphere drives the ocean. The ocean has an almost perfectly passive role, absorbing energy from the atmosphere. Concluding that the ocean has no influence on the dynamics of the atmosphere is however wrong, as demonstrated above. The amount of energy taken out of the atmosphere at its lower interface depends only slightly on the ocean dynamics, but due to the persistence of the ocean dynamics it leaves an imprint in the long-time dynamics of the atmosphere. In this respect it is difficult to dissect the atmosphere dynamics, the ocean dynamics and their interaction. One should rather see the combined system, as interactions and correlations depend on the time scale considered.

The difference of the power input estimated with the wind speeds, only, to the consistent calculation, based on the shear between the atmosphere and the ocean, is found to be small. This is different to the work by Duhaut and Straub (2006), who treated a large scale double gyre circulation. They have a constant-in-time wind velocity and the double gyre configuration that leads to a strong boundary current that separates from the boundary and penetrates into the interior of the domain with large ocean velocities of the order of $1 \mathrm{~m} / \mathrm{s}$. In our calculations periodic boundary conditions are used and no accumulation of energy at a boundary is possible. Sverdrup theory, based on the conservation of vorticity predicts velocities for an ocean subject to a constant in time large 
scale wind-forcing of only a few centimetres per second. In our calculations the forcing by the atmosphere on the ocean is (for the three lower values of the drag coefficient) constantly changing magnitude and direction, in time-andspace. This temporal and spatial incoherence in the forcing explains the feeble ocean speeds in our model and thus also the smaller differences between the correct implementation and its approximation.

For the highest drag coefficient, the atmosphere injects more energy into the ocean, this leads to stronger ocean currents. Stronger ocean currents together with the higher drag coefficient have an increased influence on the atmosphere, leading to an alignment of the instantaneous atmospheric winds with the ocean currents. This not only leads to stronger ocean currents but also to a reduction of temporal variability in the atmosphere, which furthermore reduces the temporal variability in the ocean. This positive feedback finally causes a strong oceanic current and a strong collocation of atmospheric and oceanic features. The disorder in the highest drag coefficient case is dominated by spatial disorder (quenched disorder), whereas in the three other cases, the temporal disorder prevails (annealed disorder). An astonishing result of our calculations is that the change between the two kinds of disorders seems to happen abruptly and it can not be excluded that it resembles a phase change. The transition happens when the non-dimensional stability parameter (see e.g. Chen and Jirka 1997)

$$
S=\frac{C_{d} L^{a}}{H^{a}}
$$

comparing the drag force to the advection increases towards unity. Even more striking is that the dynamics in the ocean and atmosphere does not converge to the purely periodic state imposed by the forcing but to a spatial disordered state. Such kind of behaviour is commonly observed in condensed matter physics, where it is called glass transition (see e.g. Stillinger and Debenedetti 2013 and Berthier and Biroli 2011), it occurs around a glass transition temperature, at which the internal relaxation times begin suddenly to exceed the practical measurement times and, furthermore, the state becomes protocol dependent. When the material is performing a glass transition it does not, or only very very slowly, converges to a periodic (crystalline) state. In our case the drag coefficient plays the role of the control parameter.

The quadratic dependence of the power input to the ocean is important for ocean dynamics as this small-scale energy contributes not only to the mixing in the ocean at the scale where it is injected, but will cascade to larger scales in an inverse energy cascade.

\section{Conclusions}

The majority of our results concerning the air-sea interaction were expected: the passive role of the ocean in the energy transfer; the weak dependence of the air-sea energy transfer on the ocean velocities and its increase with the drag 
coefficient; and the higher correlation of the averaged vorticities as compared to instantaneous part. The value of the present work lies in the quantification of the air sea interaction at the synoptic- and mesoscale.

The increased correlation of the vorticity between the atmosphere and the ocean (Figs. 5 and 6) when a temporal average rather than instantaneous values are considered shows, that even when such a correlation is hidden on instantaneous snapshots due to the fast turbulent dynamics of the atmosphere it emerges when averages are considered.

The most striking result is the glass transition in the dynamics of the atmosphere-ocean system when the drag coefficient is increased from $C_{d}=4 \times 10^{-4}$ to $8 \times 10^{-4}$. For the higher drag coefficient, the time variability is largely reduced and the system is in a quenched disordered state. This is similar to the what happens in a glass transition, when the temperature is reduced below critical. The consequence of this is, that time averages are different from spatial averages, such that the system is no-longer ergodic. In studies of the earth system based on observations, ergodicity is often assumed, as there is only one planet. The ergodic hypothesis has been proven not to apply to the inviscid dynamics on the $\beta$-plane and the sphere subject to strong rotation by Shepherd (1987) and questioned in other idealised models. When studies are based on laboratory experiments or numerical simulations, experiments are continued until statistical quantities have converged, using again the ergodic hypothesis. To further investigate such transition analytically and/or numerically a simpler model is necessary.

In the calculations with the high drag coefficient the high instantaneous correlation between the vorticity in the atmosphere and the ocean (Table 3 see also Fig. 2), shows that the ocean currents leave a definite imprint in the atmospheric winds leading to fine scale structures in the atmosphere. This suggest, that the fine scale structures of the ocean dynamics which have recently been exposed by fine resolution satellite observations can influence the atmospheric dynamics through momentum transfer, even when heat-fluxes are excluded. To evaluate the robustness of our results observational studies similar to Chelton et al. (2004) should also consider the correlation between persistent winds in the atmospheric boundary layer and currents in the oceanic boundary layer, at small scales.

The genesis of hurricanes is strongly influenced by air-sea interaction, mostly through the heat exchange but also by the frictionally induced convergence (Charney and Eliassen 1964). The near surface processes such as mesoscale positive vorticity anomalies are important in the bottom-up scenarios of hurricane genesis (Fang and Zhang 2008). The capture of atmospheric eddies by oceanic structures and their co-evolution can be an important ingredient in this context. To further investigate the co-evolution of atmospheric and oceanic vorticity anomalies and their statistical properties, a study with a simplified point vortex model is currently undertaken. 
References

Berthier L., Biroli, G (2011) Theoretical perspective on glass transition and amorphous materials, Rev. of Mod. Phys. 83:587-645 DOI: 10.1103/RevModPhys.83587

Charney J, Eliassen A (1964) On the growth of the hurricane depression, J Atmos Sci 21:68-75

Chelton DB, Schlax MG, Freilich MH, Milliff RF (2004) Satellite measurements reveal persistent small-scale features in ocean winds. Science 303:978-983

Courant R., Friedrichs K, Lewy H. (1928) Uber die partiellen Differenzengleichungen der mathematischen Physik Mathematische Annalen (in German), Vol 100,32-74 DOI: 10.1007/BF01448839

Duhaut HA, Straub N (2006) Wind stress dependence on ocean surface velocity: implication for mechanical energy input to ocean circulation. J Phys Oceanogr 36:202-211

Esau I (2014) Indirect air-sea interactions simulated with a coupled turbulence-resolving model, Ocean Dyn 64:689-705

Fang J, Zhang F (2010) Initial development and genesis of Hurricane Dolly (2008), J Atmos Sci 67:655-672 DOI: 10.1175/2009JAS3115.1

Ferrari R, Wunsch C (2009) Ocean circulation kinetic energy: reservoirs, sources, and sinks. Annu Rev Fluid Mech 41:253-282 DOI: 10.1146/annurev.fluid.40.111406.102139

Hogg AM, Dewar WK, Berloff P, Kravtsov S, Hutchinson DK (2009) The effects of mesoscale ocean-atmosphere coupling on the large-scale ocean circulation, J Clim 22:4066-4082 DOI: 10.1175/2009JCLI2629.1

Högström U, Rutgersson A, Sahlée E, Smedman A-S, Tihomir Hristov S, Drennan WM, Kahma KK (2013) Air-sea interaction features in the Baltic Sea and at a Pacific trade-wind site: an inter-comparison study. Boundary-Layer Meteorol 147:139-163 DOI 10.1007/s10546-012-9776-8

Moulin A, Wirth A (2014) A drag-induced barotropic instability in air-sea interaction, J Phys Oceanogr 44:733-741

Prandtl L, Schlichting H (1934) Das Widerstandsgesetz rauher Platten, Werft, Reederei, Hafen, 15:1-14

Roquet F, Wunsch C, Madec G (2011) On the patterns of wind-power input to the ocean circulation. J Phys Oceanogr 41:2328-2342 DOI: 10.1175/JPOD-11-024.1

Schlichting H, Gertsen K (2000) Boundary-Layer Theory, Springer Verlag ISBN-13: 978-3540662709.

Seo H, Murtugudde R, Jochum M, Miller AJ (2008) Modeling of mesoscale coupled ocean-atmosphere interaction and its feedback to ocean in the Western Arabian Sea, Ocean Modell 25:120-131 DOI: 10.1016/j.ocemod.2008.07.003.

Shepherd, T. G. (1987) Non-ergodicity of inviscid two-dimensional flow on a beta-plane and on the surface of a rotating sphere, J of Fluid Mechanics, 184:289-302. 
Stillinger, F.K., Debenedetti, P.G. (2013) Glass transition Thermodynamics and Kinetics, Annu. Rev. Condens. Matter Phys. 4:263-85 DOI:10.1146/annurev-conmatphys-030212-184329

Stull RB (1988) An Introduction to boundary layer meteorology. Springer, ISBN: 9027727686.

Vallis G (2006) Atmospheric and Oceanic Fluid Dynamics. Cambridge University Press, Cambridge ISBN: 0-521-84969-1 

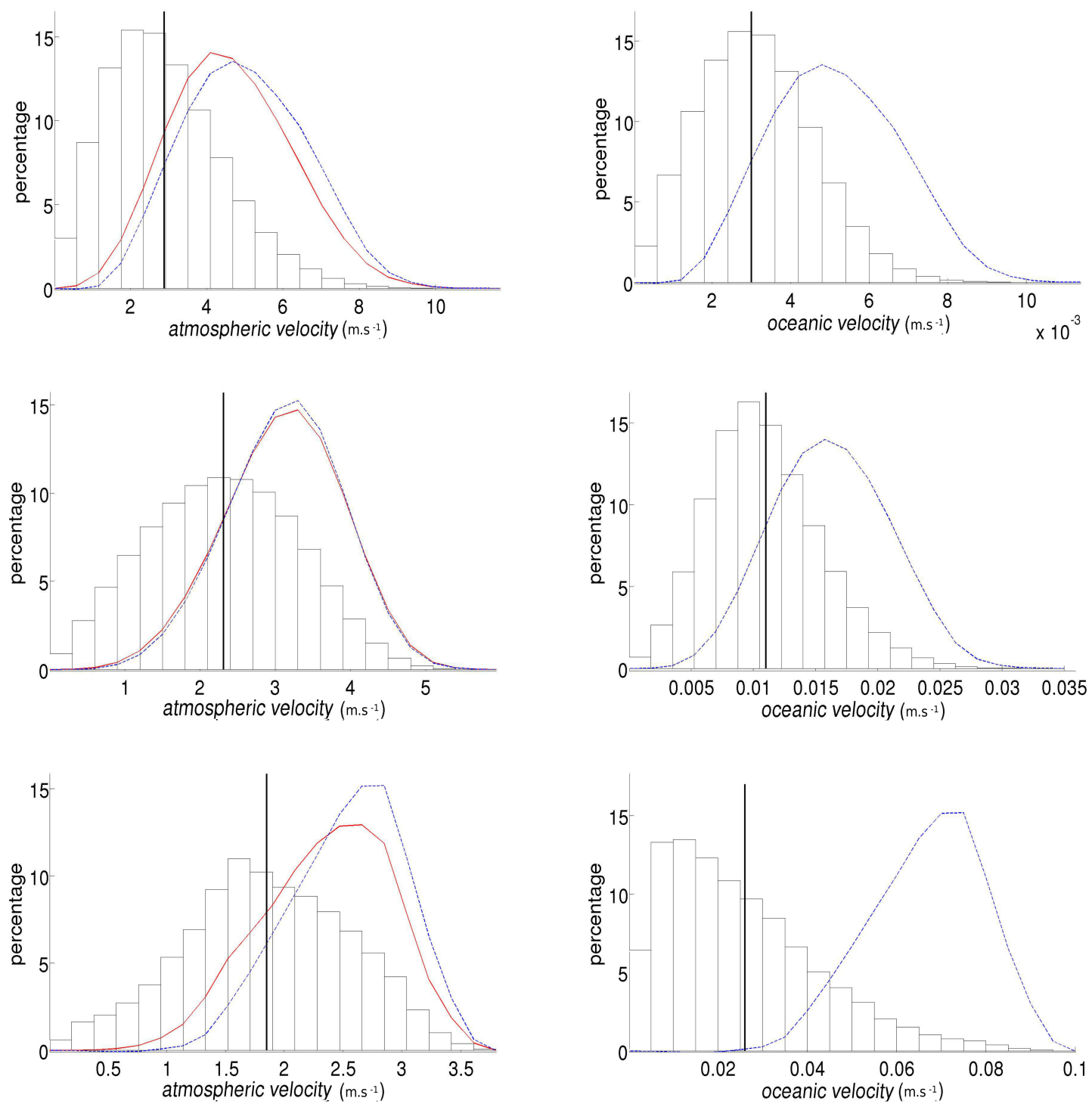

Fig. 3 Percentage of surface area for a range of speed (histogram) for the atmosphere (left row) and the ocean (right row) for different values of the drag coefficient $C_{d}=1 \times 10^{-4}$ at the top, $C_{d}=4 \times 10^{-4}$ in the middle, and $C_{d}=8 \times 10^{-4}$ at the bottom. The curves superposed give the power input calculated with the consistent formulation for the ocean (red-full) and lost for the atmosphere (blue-dashed) corresponding to the specific range of speed. 


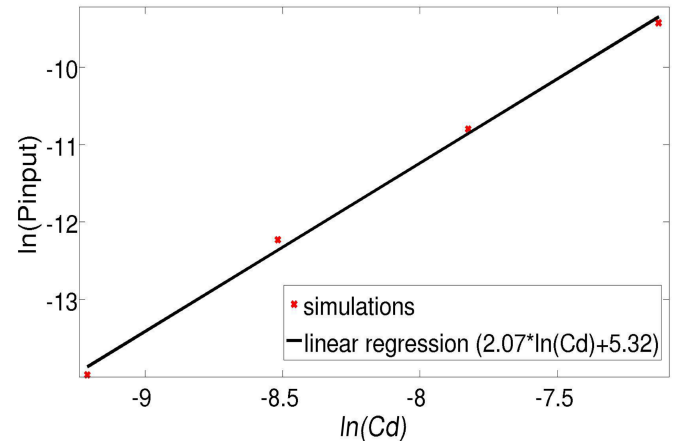

Fig. 4 Log-log plot of the mean power input (P) as a function of the drag coefficient. 

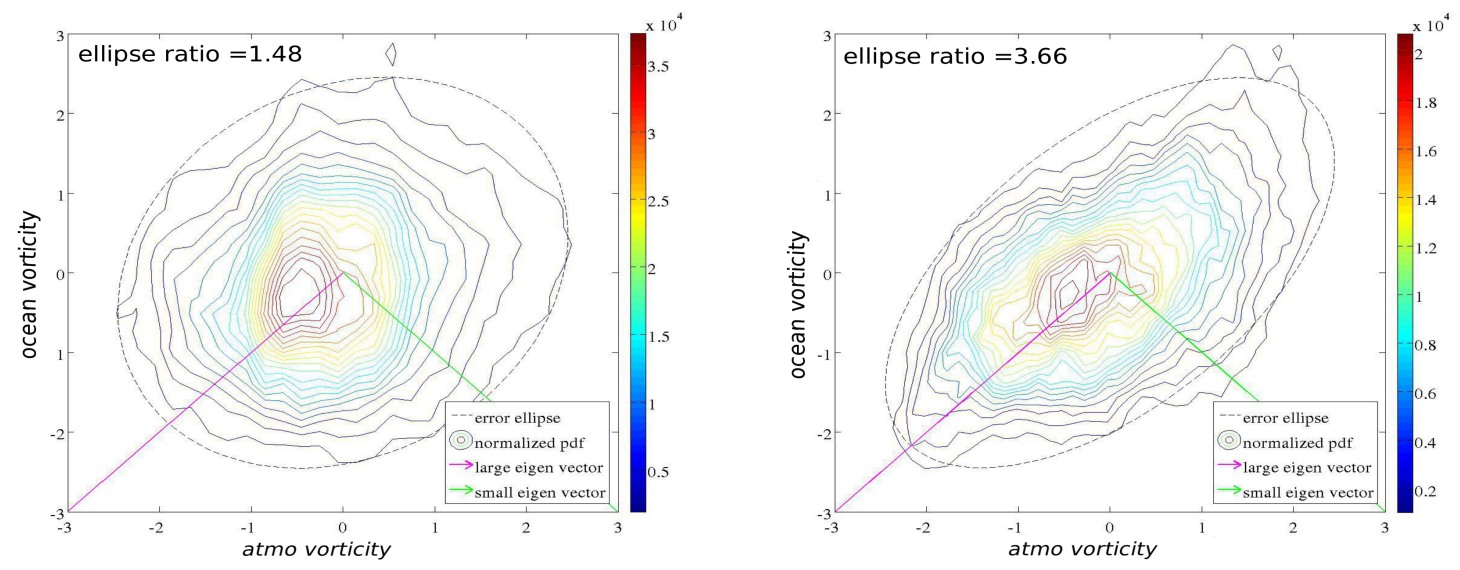

Fig. 5 Normalised probability density function for $C_{d}=1 \times 10^{-4}$ for the instantaneous snapshot after 2675 days (left) and for the mean temporal vorticity (right).
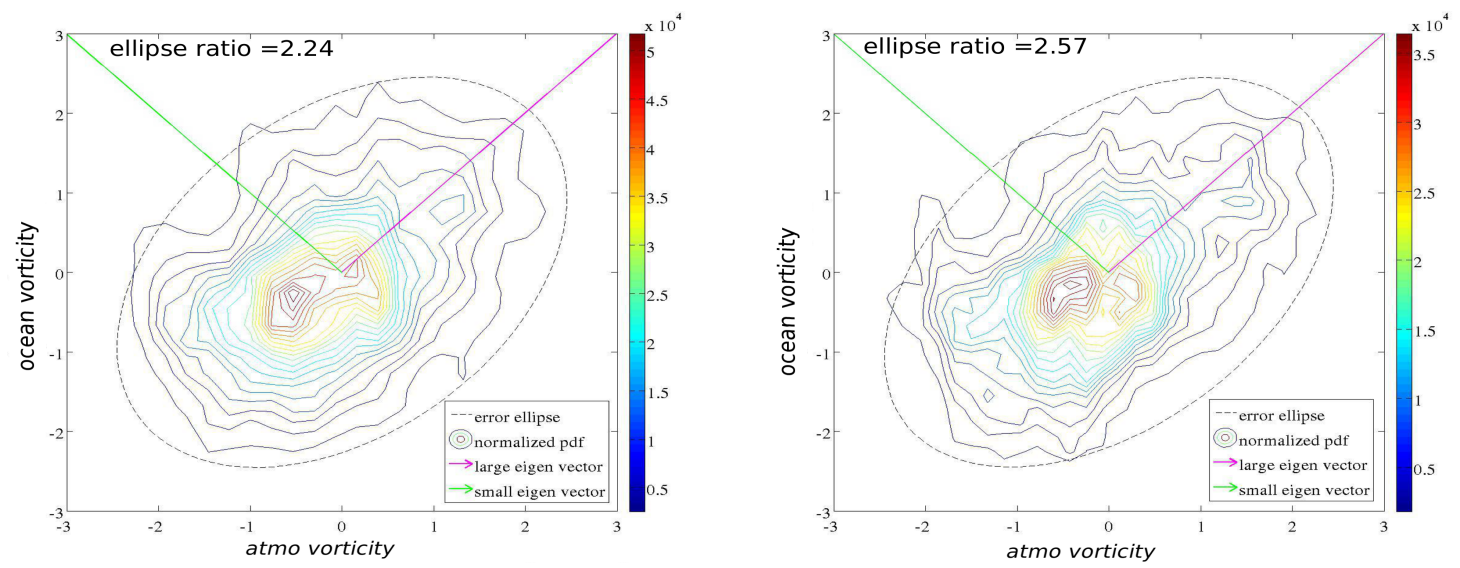

Fig. 6 Normalised probability density function for $C_{d}=8 \times 10^{-4}$ for the instantaneous snapshot after 2675 days (left) and for the mean temporal vorticity (right). 


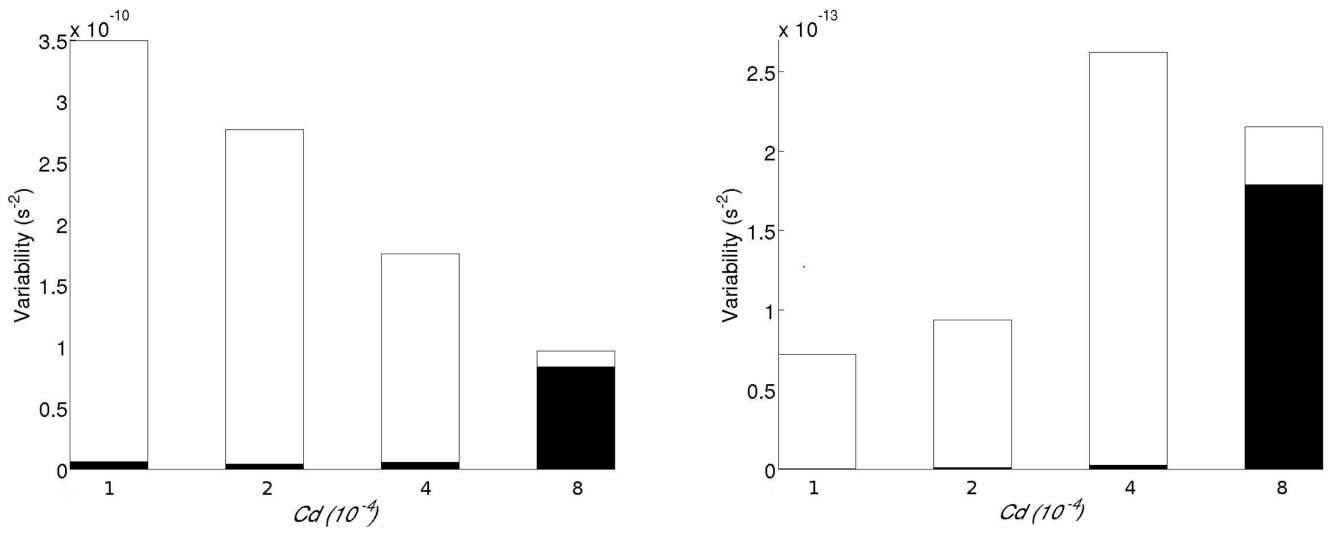

Fig. 7 Space variability (black) and time variability (white) for four values of the drag coefficient in the atmosphere (right) and in the ocean (left). For the ocean the variability are multiplied by 100 for the three lower drag coefficients. 